ARTICLE

Received 22 Feb 2014 | Accepted 11 Apr 2014 | Published 16 May $2014 \quad$ DOI: 10.1038/ncomms4872

\title{
Emergent properties of the interferon-signalling network may underlie the success of hepatitis $C$ treatment
}

\author{
Pranesh Padmanabhan ${ }^{1}$, Urtzi Garaigorta ${ }^{2} \&$ Narendra M. Dixit ${ }^{1}$
}

Current interferon alpha-based treatment of hepatitis $\mathrm{C}$ virus (HCV) infection fails to cure a sizeable fraction of patients treated. The cause of this treatment failure remains unknown. Here using mathematical modelling, we predict treatment failure to be a consequence of the emergent properties of the interferon-signalling network. HCV induces bistability in the network, creating a new steady state where it can persist. Cells that admit the new steady state alone are refractory to interferon. Using a model of viral kinetics, we show that when the fraction of cells refractory to interferon in a patient exceeds a critical value, treatment fails. Direct-acting antivirals that suppress HCV replication can eliminate the new steady state, restoring interferon sensitivity and improving treatment response. Our study thus presents a new conceptual basis of HCV persistence and treatment response, elucidates the origin of the synergy between interferon and direct-acting antivirals, and facilitates rational treatment optimization.

\footnotetext{
${ }^{1}$ Indian Institute of Science, Department of Chemical Engineering, Bangalore 560012, India. ${ }^{2}$ The Scripps Research Institute, Department of Immunology and Microbial Science, La Jolla, California 92037, USA. Correspondence and requests for materials should be addressed to N.M.D.

(email: narendra@chemeng.iisc.ernet.in).
} 
epatitis $\mathrm{C}$ virus (HCV) infection is spread over $130-170$ million people worldwide ${ }^{1}$. Pegylated interferon- $\alpha$ (IFN$\alpha$ ), in combination with ribavirin, forms the mainstay of the current treatment for HCV infection. The combination (PR), however, elicits a sustained virological response (SVR) in only about $50 \%$ of HCV genotype 1 infected patients treated ${ }^{2}$. Several factors that correlate with treatment response have been identified, including HCV genotype, pre-treatment viral load and single-nucleotide polymorphisms (SNPs) in the IFN- $\lambda$ locus ${ }^{3,4}$. The cause of treatment failure, however, remains poorly established.

IFN- $\alpha$ belongs to a family of cytokines produced endogenously in response to viral infection ${ }^{5,6}$. Through a complex series of signalling events involving the JAK-STAT pathway, stimulation by IFN results in the expression of several hundred IFNstimulated genes (ISGs) which together induce an antiviral state in cells and control infection ${ }^{6}$. With $\mathrm{HCV}$, however, a vast majority $(\sim 70-80 \%)$ of infected individuals are unable to clear the infection spontaneously and become chronically infected ${ }^{6,7}$. PR treatment can compensate for suboptimal immune control of infection. ISG expression following the onset of treatment was enhanced substantially over the pre-treatment level in the livers of $\mathrm{HCV}$ patients who responded to $\mathrm{PR}^{8-10}$. (Ribavirin alone elicits no $\mathrm{SVR}^{11}$, but improves response in combination with IFN $^{12}$, suggesting that ribavirin potentiates the anti-HCV activity of IFN $^{10,13-15}$.) Surprisingly, however, in the livers of nonresponders to $\mathrm{PR}$ treatment, pre-treatment ISG expression was higher than in responders and did not increase significantly following the onset of treatment ${ }^{8-10}$.

Non-responsiveness to $\mathrm{PR}$ was eliminated in part by the recently approved direct-acting antiviral agents (DAAs) telaprevir and boceprevir, each of which in conjunction with PR yielded SVR rates of over $70 \%$ in previously untreated HCV genotype 1 infected patients ${ }^{2,16}$. Because telaprevir and boceprevir monotherapy fail owing to the emergence of drug resistance, one hypothesis for the success of the triple combination is that DAAs act on drug sensitive strains whereas PR control resistant strains ${ }^{17}$. Paradoxically, however, although the DAAs alone elicited no SVR, the triple combination induced SVR in $40 \%$ of previous null responders to $\mathrm{PR}$, who showed no significant decline in viral levels during PR treatment indicating the near absence of IFN activity ${ }^{18,19}$. DAAs thus appear to synergize with IFN and help overcome non-responsiveness to PR.

In two recent pioneering studies, poor response following highpre-treatment ISG expression has been suggested to arise from upregulation, associated with persistent IFN signalling, of immunosuppressive molecules such as interleukin-10 and programmed death-ligand 1 (PD-L1), which leads to T-cell exhaustion and viral persistence ${ }^{20,21}$. These studies mark a breakthrough in our understanding of chronic viral infections and recommend, counter intuitively, suppression of IFN signalling to avert $\mathrm{T}$-cell exhaustion and elicit control of chronic infection. Two key questions, however, remain unanswered. When does HCV infection lead to high (pretreatment) ISG expression and therefore poor-treatment response? How, despite high-exogenous IFN addition, which would ensure continued immunosuppression following the onset of treatment, do DAAs improve treatment response in combination with PR? Mechanisms in addition to IFNassociated immunosuppression thus appear to contribute to $\mathrm{HCV}$ persistence and treatment response.

Previous studies have identified several mechanisms by which $\mathrm{HCV}$ evades IFN activity: $\mathrm{HCV}$ can suppress endogenous IFN production, inhibit ISG expression by interfering with the JAK-STAT pathway, induce a block in the translation of ISG mRNA, and/or inhibit ISG effector functions ${ }^{6,22}$. At the same time, key ISGs involved in the control of HCV have been identified and in some cases their mode of action elucidated ${ }^{23-27}$. These specific interactions between $\mathrm{HCV}$ and components of the IFN network have unravelled the many fronts of the battle between HCV and our innate immune system. The outcome of this battle, however, has been difficult to predict: the specific interactions prove inadequate for distinguishing between nonresponders and responders to $\mathrm{PR}$ or explaining the observed synergy between DAAs and $\mathrm{PR}$, precluding rational strategies for improving treatment response.

In the present study, we explore the possibility that responsiveness to IFN is not a consequence of specific molecular interactions between individual components of the IFN system and HCV alone, but of emergent, systems-level properties of the IFN signalling network interacting with HCV. The IFN network is designed to suppress $\mathrm{HCV}$ upon stimulation. $\mathrm{HCV}$, on the other hand, interferes with IFN signalling and compromises its effector functions. Non-responsiveness to IFN may thus be a manifestation of the state to which the IFN system is driven as a consequence of these competing interactions. Further, although DAAs directly target viral (or host) proteins, their action may, at the systems level, weaken the influence of HCV on the IFN network and drive the system to an alternative, responsive state. To test this hypothesis, we construct a mathematical model of the IFN signalling network in the presence of HCV and elucidate its systems-level properties. We find intriguingly that HCV induces bistability in the network, causing the emergence of a new steady state where $\mathrm{HCV}$ persists. Accounting for this bistability, we develop a mathematical model of viral kinetics and deduce a criterion for treatment response. Finally, we find that DAAs can eliminate this bistability and improve treatment response.

\section{Results}

Model of the IFN signalling network in the presence of HCV. We considered the intracellular IFN signalling network in the presence of HCV (Fig. 1). Upon infection, HCV replicates inside the cell producing copies of positive and negative strands of RNA, the latter presumably existing as double-stranded RNA (dsRNA). In the presence of IFN, ISGs are transcribed to produce ISG mRNA. The translation of ISG mRNA yields ISG proteins, which control HCV by inhibiting different steps of the HCV life cycle and/or lowering the stability of HCV $\mathrm{RNA}^{23-27}$. Of the ninety ISGs expressed in response to IFN (type I) stimulation, seven were recently found to exhibit significant activity against $\mathrm{HCV}^{24}$. HCV can evade IFN action by several mechanisms including lowering global translation in the cell via protein kinase $\mathrm{R}$ $(\mathrm{PKR})^{28}$. The latter mechanism has been shown in recent in vitro studies to facilitate $\mathrm{HCV}$ persistence despite strong IFN treatment ${ }^{28}$, postulated to be the predominant mode of the interference of IFN signalling by $\mathrm{HCV}$ in vivo ${ }^{29}$, and may represent a key pathway underlying the resistance of HCV to $\mathrm{IFN}^{30}$. We therefore focused here on the HCV-mediated block in the translation of ISG mRNA via PKR. (We also considered other mechanisms of the interference of IFN signalling by HCV and found similar results; see Discussion.) Initiation of translation depends on the abundance of the eIF $2 \alpha$-guanosine triphosphate (GTP) complex. eIF2 $\alpha$-GTP gets hydrolyzed to eIF2 $\alpha$-guanosine diphosphate (GDP) during translation ${ }^{31}$. The recycling of eIF2 $\alpha-$ GDP to eIF2 $\alpha-$ GTP is mediated by another protein eIF2B ${ }^{32}$. $\mathrm{HCV}$ induces PKR dimerization, presumably by the binding of $\mathrm{HCV}$ double-stranded RNA to $\mathrm{PKR}^{33,34}$. PKR dimers undergo autophosphorylation $^{34}$. Phosphorylated PKR catalyzes the phosphorylation of eIF2 $\alpha-$ GDP. Phosphorylated eIF2 $\alpha-$ GDP does not undergo GDP to GTP exchange and has an increased affinity for eIF2 $\mathrm{B}^{31,32}$. eIF2B is present in low abundance 


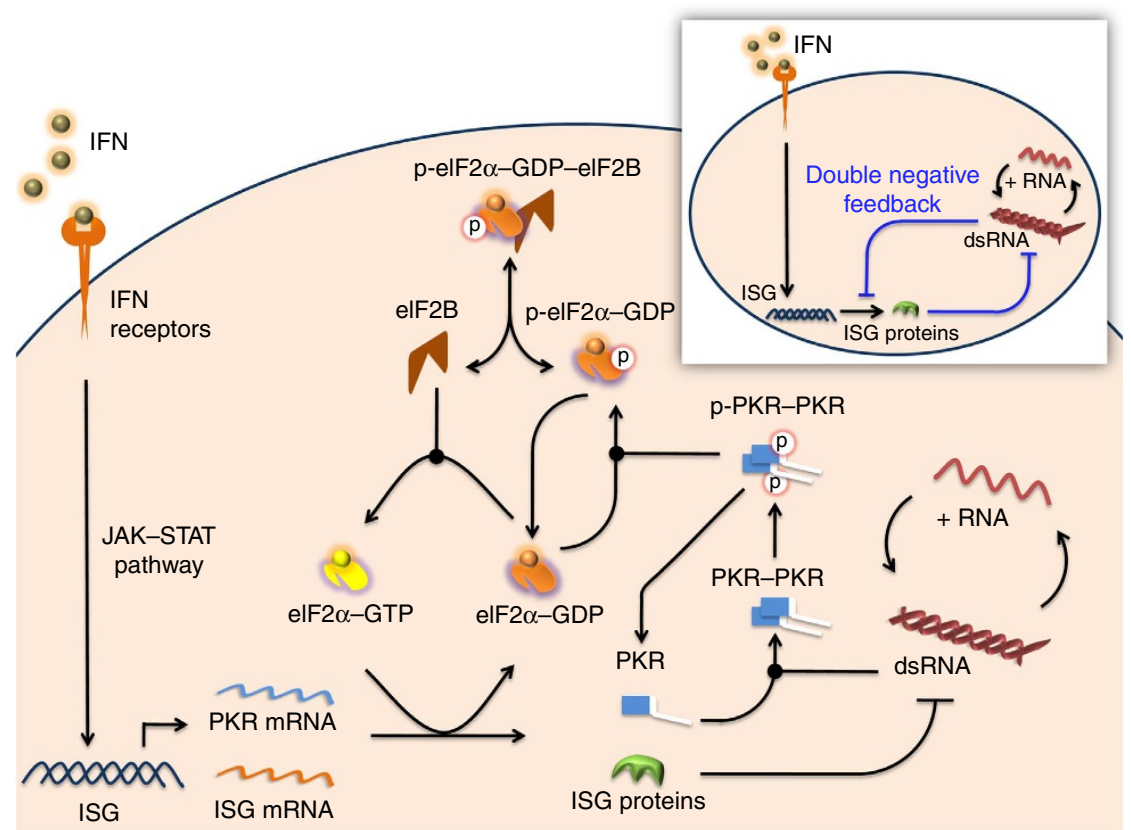

Figure 1 | Schematic of the IFN signalling network in the presence of HCV. Following exposure to IFN, signalling via the JAK-STAT pathway triggers ISG expression, which lowers the HCV RNA level inside a cell. HCV catalyzes PKR dimerization and autophosphorylation. Phosphorylated PKR phosphorylates elF2 $\alpha-G D P$, which sequesters elF2B and lowers translation of ISG mRNA. Inset: schematic of the double-negative feedback in the network owing to the suppression of HCV by ISG proteins and of ISG proteins by HCV.

compared with eIF $2 \alpha^{31}$. Thus, phosphorylated eIF2 $\alpha-$ GDP sequesters eIF2B, which further reduces the eIF2 $\alpha-$ GTP level, blocking translation. HCV, however, continues to replicate as its translation is less sensitive to eIF $2 \alpha-G_{T} 28,35-37$. The HCV-PKR interaction thus contains key elements of the suppression of IFN activity by HCV as well as the control of HCV by IFN, capturing the essence of the battle between HCV and IFN at the systems level. We modelled the network using a system of coupled differential equations (Supplementary Note 1) and elucidated its emergent properties.

Bistability in the IFN signalling network induced by HCV. The network exhibits double-negative feedback: ISG proteins control $\mathrm{HCV}$, whereas HCV controls ISG mRNA translation. Doublenegative feedback with ultra-sensitivity (nonlinearity) can give rise to bistability ${ }^{38}$, observed in several signalling and transcription networks ${ }^{38-42}$. To test the existence of bistability, we identified the steady states of the network as follows. We predicted first the dependence of the steady state ISG protein level on the HCV RNA level (red line in Fig. 2). Here the doublestranded HCV RNA level was fixed, thus eliminating the negative feedback of ISG on HCV, and the system solved for steady state. This process was repeated for different values of the doublestranded HCV RNA level. Similarly, we predicted the dependence of the steady state HCV RNA level on the ISG protein level (blue line in Fig. 2), by suppressing the negative feedback of HCV on ISG. When both the feedback loops are active, the intersection points $Q_{1}, Q_{2}$ and $Q_{3}$ represent the steady states of the system (Fig. 2). At $Q_{1}$, the HCV RNA level is high and the ISG protein level low, marking HCV persistence, whereas at $Q_{3}, H C V$ RNA is absent and the ISG protein level is high, indicating HCV clearance. $Q_{1}$ and $Q_{3}$ are the two stable steady states of the system. $Q_{2}$, where HCV RNA and ISG protein levels are intermediate, is an unstable steady state, in that small perturbations get amplified and drive the system away from $Q_{2}$.

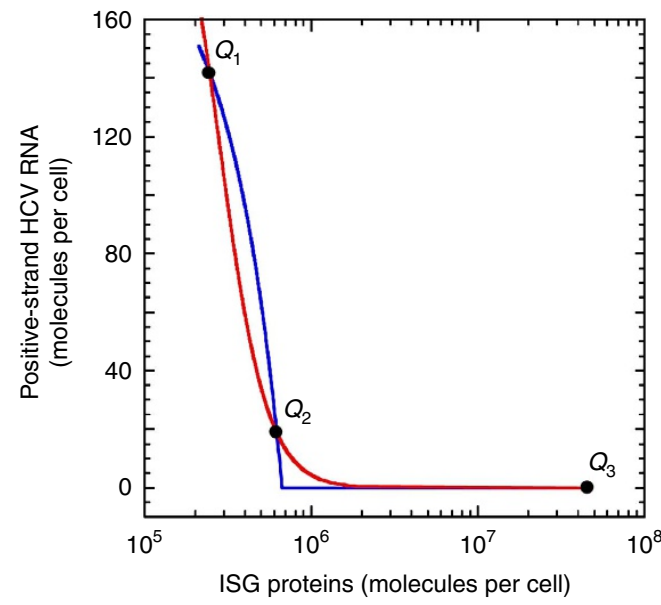

Figure 2 | HCV-induced bistability in the IFN signalling network. Model predictions of concentration-effect curves showing steady state positivestrand HCV RNA levels for fixed total ISG protein levels (blue line) and total ISG protein levels for fixed double-stranded HCV RNA levels (red line). Both curves are drawn with positive-strand HCV RNA levels on the $y$ axis and ISG protein levels on the $x$ axis. The ISG proteins include the seven ISGs, namely, IFIT3, IFITM1, IFITM3, PLSCR1, TRIM14, Viperin and RNase L, identified recently to have anti-HCV activity ${ }^{24}$ (Supplementary Note 1). The intersections of the curves mark the three steady states, $Q_{1}, Q_{2}$ and $Q_{3}$, of the network. Parameters employed are listed in Supplementary Tables 1-3.

$Q_{2}$, thus acts as a threshold determining which of the two stable steady states the system eventually attains.

Bistability in in vitro experiments and model validation. Evidence of this threshold and the underlying bistability is found in recent in vitro experiments ${ }^{28}$. In the latter experiments, cells were treated with IFN before or at different times post infection 

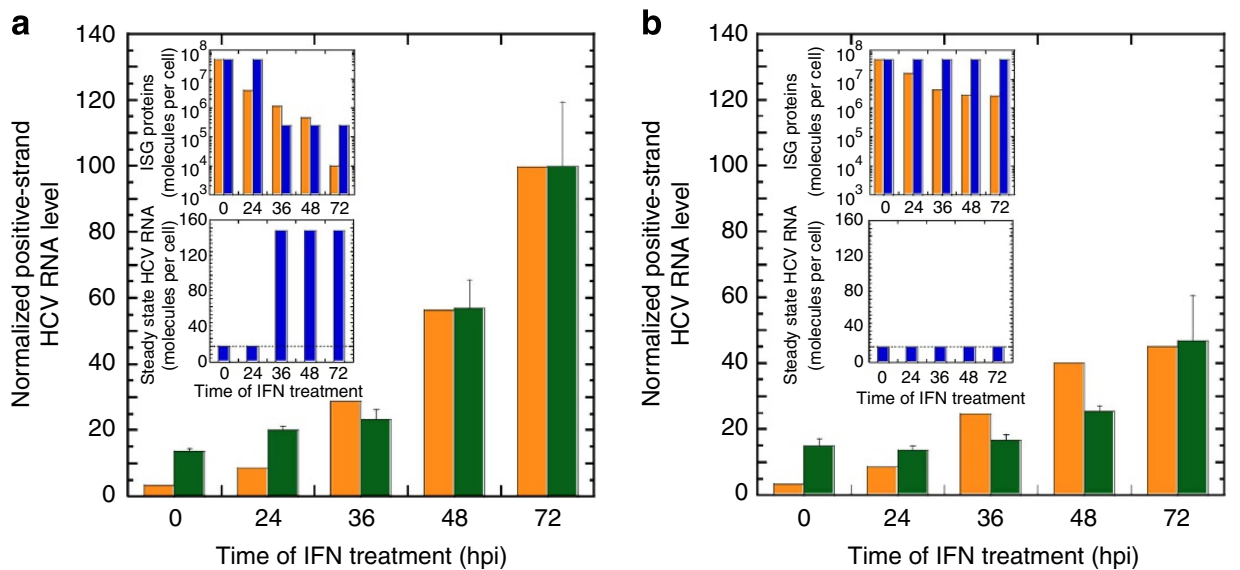

Figure 3 | Model validation against in vitro data. Normalized positive-strand HCV RNA levels predicted by our model (orange) and measured previously in in vitro experiments ${ }^{28}$ (green) at $20 \mathrm{~h}$ post IFN treatment $\left(100 \mathrm{IU} \mathrm{ml} \mathrm{m}^{-1}\right.$ ) following different specified times (hours post infection) with HCV. Error bars represent standard deviations from triplicates. Lower inset: model predictions of corresponding HCV RNA levels achieved at steady state post IFN treatment. Upper inset: model predictions of corresponding ISG protein levels at $20 \mathrm{~h}$ (orange) and at steady state (blue) post IFN treatment. (a) normal cells and (b), PKR-silenced cells (60\% reduction in PKR mRNA transcription rate). The dashed line marks zero. Normalization was done with the positivestrand HCV RNA level in cells without IFN treatment. Parameters employed are listed in Supplementary Tables 1-3.

and HCV RNA and ISG protein levels were measured $20 \mathrm{~h}$ post IFN treatment ${ }^{28}$. When treated up to $24 \mathrm{~h}$ post infection, phosphorylated PKR levels were low, ISG protein levels were high and HCV RNA levels suppressed. When treated $\geq 48 \mathrm{~h}$ post infection, phosphorylated PKR levels were high, ISG protein levels low and HCV RNA levels approached that of IFN untreated cells. Using parameter values obtained independently (Supplementary Note 2, Supplementary Tables 1-3), our model captured the above observations quantitatively (Fig. 3a). According to our model, following infection, the HCV RNA level gradually increases inside cells (Supplementary Fig. 1A); endogenous IFN production is not expected in the in vitro cell-culture infection system $^{43}$. If IFN is added when the HCV RNA level is below the threshold corresponding to $Q_{2}$, the system drops to $Q_{3}$, where $\mathrm{HCV}$ is controlled. In contrast, if IFN is added after the HCV RNA level crosses the threshold, the system reaches $Q_{1}$, where $\mathrm{HCV}$ persists. The switch in the response of the system based on the time of IFN addition is thus a signature of bistability. Indeed, silencing PKR, which suppresses one of the feedback loops, eliminated this signature in the experiments and in our model predictions (Fig. 3b). Further, our model mimicked all the observations of the latter in vitro study ${ }^{28}$ (Supplementary Note 3, Supplementary Figs 1-3), giving us confidence in its ability to accurately unravel the behaviour of the underlying network.

Diversity of the cellular response to IFN. We found that the network exhibited bistability over several-fold changes in the values of several parameters (Supplementary Fig. 4). Global sensitivity analysis yielded parameters to which the systems-level properties of the network were sensitive (Supplementary Note 4, Supplementary Fig. 4). Bistability was lost when parameter values tilted the competition between HCV and IFN heavily in favour of either one. For instance, when the repression coefficients for the control of HCV replication by ISGs were decreased, IFN effectiveness increased and the system admitted the steady state $Q_{3}$ alone, where $\mathrm{HCV}$ is eliminated. When the repression coefficients were increased, IFN effectiveness decreased and the system admitted $Q_{1}$ alone, where HCV thrives (Fig. 4a).

It follows that intrinsic variations across cells, for example, in ISG expression ${ }^{44,45}$ or the expression of host protease and other
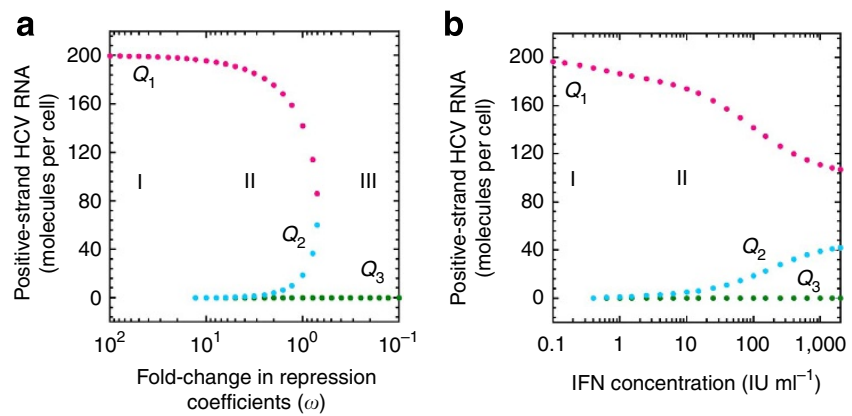

Figure 4 | Hysteresis and distinct cellular responses to IFN. Steady states of the network, $Q_{1}$ (pink), $Q_{2}$ (cyan) and $Q_{3}$ (green) over a range of values of (a), the repression coefficients for the control of HCV replication by ISGs ( $\omega$ denotes the fold-change in the repression coefficients of all ISGs relative to their baseline values in Supplementary Table 3), and (b), the IFN level. The three distinct cellular response phenotypes (see text) are marked. Region I admits $Q_{1}$ alone, region II both $Q_{1}$ and $Q_{3}$ and region III $Q_{3}$ alone. In b, region III does not occur within the range of IFN levels considered. Other parameters employed are listed in Supplementary Tables 1-3.

enzymes that affect HCV replication, would yield three distinct cellular phenotypes based on the response to a given level of IFN stimulation, viz., cells that admit $Q_{1}$ alone, $Q_{3}$ alone or both $Q_{1}$ and $Q_{3}$. Cells that admit $Q_{1}$ alone would allow HCV persistence even with IFN stimulation. Cells that admit $Q_{3}$ alone would clear HCV following IFN stimulation. Cells that admit both $Q_{1}$ and $Q_{3}$ would either allow HCV persistence or clear HCV depending on the time of IFN addition, as illustrated above. Increasing the level of IFN stimulation could convert a cell that admits $Q_{1}$ alone to one that admits both $Q_{1}$ and $Q_{3}$ and further to one that admits $Q_{3}$ alone. Decreasing the level of IFN stimulation could reverse these transitions. Bistable systems, however, display hysteretic behaviour: the level of IFN stimulation required to drive a cell from $Q_{1}$ to $Q_{3}$ would be larger than the level at which the cell transitions from $Q_{3}$ to $Q_{1}$. The former level may lie beyond experimentally/clinically employed levels of IFN $^{28}$ (Fig. 4b). Thus, cells in the state $Q_{1}$ may allow HCV persistence despite seemingly high levels of IFN stimulation and are therefore predicted to be 
refractory to IFN. In contrast, cells in the state $Q_{3}$ clear $\mathrm{HCV}$ infection with much lower levels of IFN stimulation following exposure to HCV (Fig. 4b). The latter cells are thus sensitive to IFN. How an HCV-infected individual responds to IFN-based treatment would thus be determined by the distribution of cells in the individual across the different IFN response phenotypes.

Viral kinetics and treatment outcome. To predict the ensuing viral kinetics and treatment outcome, we advanced the basic model of viral kinetics in vivo ${ }^{14,46-49}$ (Supplementary Note 5) by explicitly considering cell subpopulations with the three distinct IFN response phenotypes (Fig. 5a). We divided target cells into subpopulations $T_{i}$ with $i=1,2$ and 3 representing cells that admit $Q_{1}$ alone, both $Q_{1}$ and $Q_{3}$, and $Q_{3}$ alone, respectively, at the level of IFN exposure employed in the treatment; because ribavirin potentiates IFN activity, the subpopulations may be defined based on the combined activity of PR. Correspondingly, infected cells are divided into subpopulations $I_{1}, I_{2}$ and $I_{3}$. From the above analysis, it follows that cells $T_{1}$ remain susceptible to infection and are refractory to IFN. IFN does not inhibit de novo infection in this subpopulation. Cells $T_{2}$ are sensitive to IFN, as IFN exposure before HCV infection drives these cells to the state $Q_{3}$ and prevents de novo infections. Cells $T_{3}$ are similarly protected from new infections. Cells $I_{1}$ are again refractory to IFN and continue viral production. Cells $I_{2}$ are infected before IFN exposure and thus attain the state $Q_{1}$ following IFN exposure and continue to produce virions. Cells $I_{3}$ admit $Q_{3}$ alone and are cured following exposure to IFN.

The resulting viral kinetics typically follows a biphasic decline (Fig. 5b). We estimate with our model that the viral load at the end of the first phase is $\sim V_{0}\left(1-\phi_{3}\right)$, where $V_{0}$ is the baseline viral load and $\phi_{3}$ is the pre-treatment frequency of IFN-sensitive cells (Supplementary Note 6, Supplementary Fig. 5A). The extent of
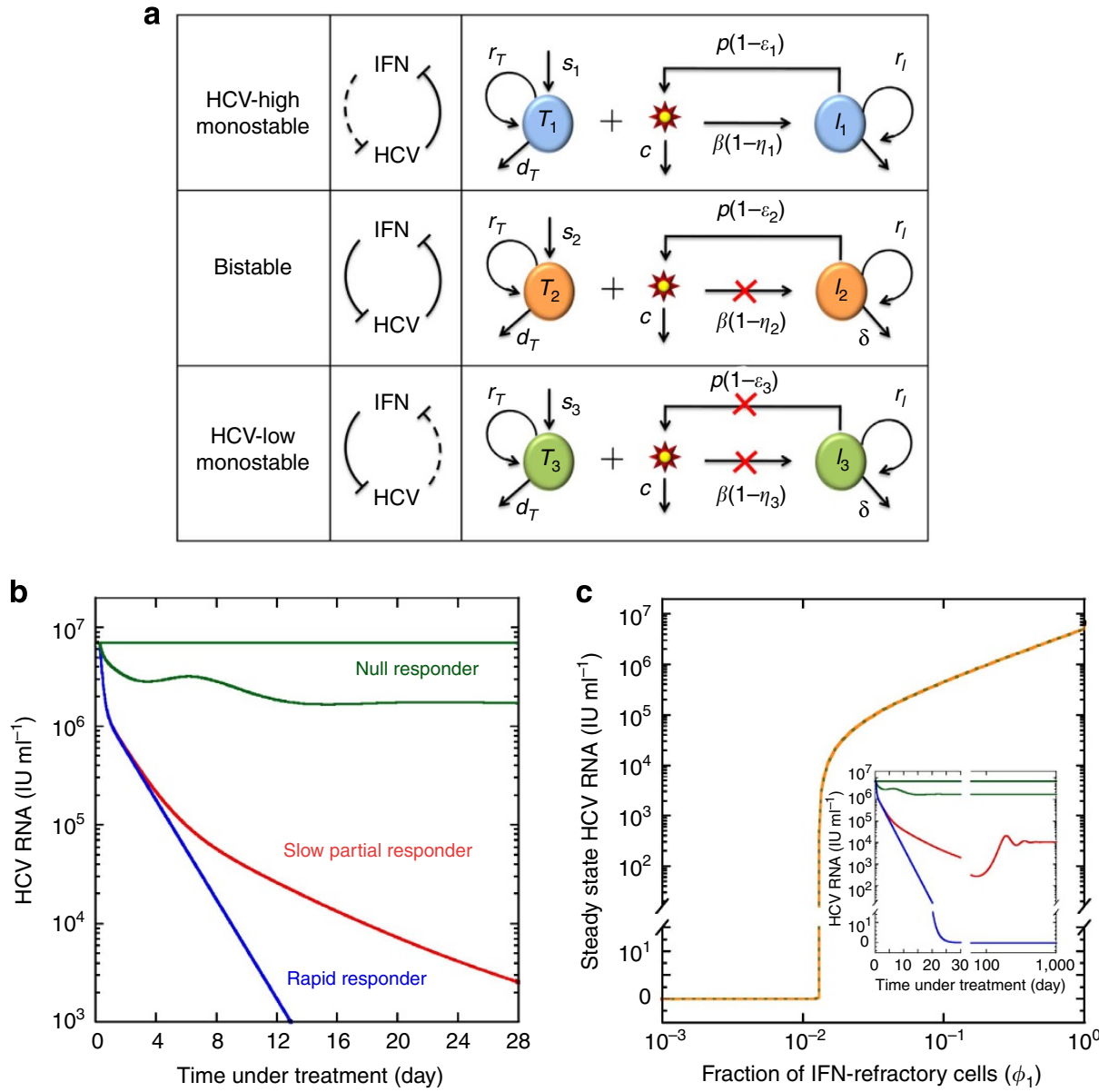

Figure 5 | Viral kinetics following IFN treatment. (a) Schematic of viral kinetics incorporating distinct cellular responses to IFN. Top: cells that have poor IFN control of HCV (left) admit $Q_{1}$ alone and are refractory to IFN (right). Middle: cells that have intermediate IFN control of HCV and vice versa (left) admit both $Q_{1}$ and $Q_{3}$ and thus continue viral production when infected but are protected from new infections by IFN (right). Bottom: cells that have strong IFN control of HCV (left) admit $Q_{3}$ alone and are sensitive to IFN (right). Target cells in the three subpopulations, $T_{i}$, where $i=1,2$ and 3 , are produced at the rates $s_{i}$, proliferate with the rate constant $r_{T}$, die with the rate constant $d_{T}$ and are lost owing to de novo infection with the rate constant $\beta$. The corresponding infected cells $l_{i}$ proliferate with the rate constant $r_{l}$ and die with the rate constant $\delta$. Free virions are produced by infected cells with the rate constant $p$ and are cleared with the rate constant $c$. The de novo infection rate and virion production rate were assumed to be blocked with efficacies $\eta_{i}$ and $\varepsilon_{i}$ in the respective subpopulations owing to PR treatment. (b) Model predictions of different viral response patterns following IFN treatment for different distributions of cells in the three IFN response phenotypes: $\phi_{1}=1, \phi_{2}=0$, and $\phi_{3}=0$ (green top); $\phi_{1}=0.35, \phi_{2}=0.35$, and $\phi_{3}=0.3$ (green bottom); $\phi_{1}=0.015, \phi_{2}=0.185$, and $\phi_{3}=0.8$ (red); and $\phi_{1}=0, \phi_{2}=0.2$, and $\phi_{3}=0.8$ (blue). (c) Steady state viral levels following IFN treatment as a function of $\phi_{1}$ when $\phi_{2}=\phi_{3}$ (solid line) and $\phi_{2}=0.5 \phi_{3}$ (dashed line) (the two curves overlap) and (Inset) long-term virological response corresponding to the predictions in b. Parameters: $V_{0}=7 \times 10^{6} \mathrm{IU} \mathrm{ml}-1, \beta=3 \times 10^{-7} \mathrm{ml}$ per virions per day. Other parameter values employed are listed in Supplementary Table 4. 
the first phase decline is thus determined by the fraction of cells cured by IFN. The second phase slope depends on the dynamics of the infected cells. Whereas cells $I_{2}$ and $I_{3}$ decline at the death rate $\delta$-de novo infection is blocked by IFN in these subpopulations and infected cells are expected not to proliferate significantly ${ }^{48}$ (Supplementary Table 4 ) -the loss of cells $I_{1}$ is compensated at least in part by the de novo infection of cells $T_{1}$, refractory to IFN (Supplementary Note 5, Supplementary Fig. 5B). The early second phase slope estimated using our model is $\sim \delta\left(1-\phi_{1}\right)$. The second phase slope thus decreases as the pre-treatment frequency of IFN-refractory cells, $\phi_{1}$, increases (Supplementary Note 6, Supplementary Fig. 5 C). By varying the frequencies $\phi_{1}, \phi_{2}$ and $\phi_{3}$, our model reproduced all the patterns of viral kinetics observed in patients (Fig. 5b).

The second phase slope in our model depends on $\phi_{1}$. Indeed, we find that when $\phi_{1}$ is below a critical value, $\phi_{1}^{c}$, viral clearance is achieved (Fig. 5c). This critical value does not depend on $\phi_{2}$ and $\phi_{3}$ because following treatment, cells $T_{2}$ and $T_{3}$ do not acquire new infections and cells $I_{2}$ and $I_{3}$ are eventually lost owing to cell death even when IFN fails to cure them. IFNrefractory cells, $T_{1}$, remain susceptible to new infections even during treatment and thus determine treatment outcome. When their number is sufficiently small, viral production from infected cells is inadequate to replenish the loss of infected cells owing to cell death (akin to the basic reproductive number being below unity $^{50}$ ), resulting in the eventual clearance of infection. Thus, the success of IFN treatment depends primarily on the size of the IFN-refractory subpopulation. The larger the latter subpopulation, the smaller the second phase slope, thus presenting an explanation of the strong correlation observed between the second phase slope and treatment response ${ }^{46,51,52}$.

Comparison with patient data. We next applied our model to analyze data of viral kinetics obtained previously from patients under IFN monotherapy ${ }^{46}$. Our model provided excellent fits to the data (Fig. 6a, Supplementary Fig. 6, Supplementary Tables 5 and 6). In agreement with our prediction above, we found from the fits that $\bar{\phi}_{1}$, an upper bound on $\phi_{1}$ obtained by letting $\phi_{2}=0$, increased from 0.01 for a typical rapid responder, to 0.11 for a slow partial responder and 0.37 for a null responder. Similarly, patients with known favourable haplotypes based on SNPs in the IFN- $\lambda$ locus had significantly lower $\phi_{1}$ on an average than patients with unfavourable haplotypes (Supplementary Fig. 6, Supplementary Table 6). Finally, we estimated $\widehat{\phi}_{1}$ for a large set of patients under IFN monotherapy ${ }^{51,53}$ and found it to be negatively correlated with the second phase slope (Fig. 6b), reiterating the dependence of the second phase slope and hence treatment outcome on the frequency of IFN-refractory cells.

Pre- and post-treatment ISG expression and response to IFN. We next examined how treatment response correlated with preand post-treatment ISG expression. The pre-treatment frequency of cells refractory to IFN would depend on the level of endogenous IFN production following infection as well as the strength of the effector function of ISGs, or, more generally, the effector function of the IFN network. IFN production stimulates the IFN network, whose effector function determines the propensity to clear HCV. Four possibilities thus arise (Fig. 7).

When endogenous IFN production is low (for example, owing to HCV-mediated cleavage of mitochondrial antiviral signaling protein (MAVS ${ }^{43,54}$ ) and IFN effector function potent, infection is established because of inadequate stimulation of the IFN network. ISG levels are predicted to be low in such individuals before treatment (Fig. 7a). Exogenous IFN addition during
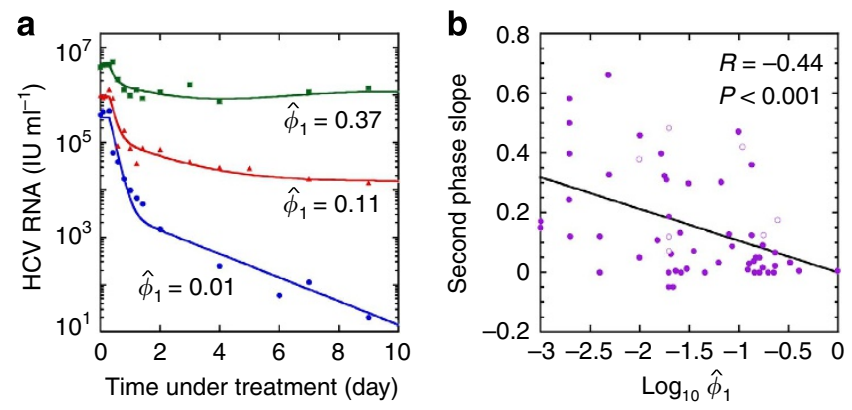

Figure 6 | Analysis of patient data. (a) Fits of model predictions of viral load decay following IFN treatment (lines) to previous patient data 46 (symbols), where the decay patterns mimic typical null responders (green), slow partial responders (red) and rapid responders (blue). We fixed known model parameter values from the literature $46,48,49$ (Supplementary

Table 4). We let inter-patient variations be determined by the parameters $\beta$ and $V_{0}$ and the distribution of cells in the three IFN response phenotypes, $\phi_{1}, \phi_{2}$ and $\phi_{3}$. We recognized that $\phi_{3}$ is determined by the viral load decline in the first phase (see text). The available second phase data did not allow the estimation of both $\phi_{1}$ and $\phi_{2}$ uniquely. We therefore assumed $\phi_{2}=0$ and estimated $\widehat{\phi}_{1}$ as the corresponding upper bound on $\phi_{1}$. We thus let $\beta$, $V_{0}$ and $\widehat{\phi}_{1}$ be adjustable parameters. The resulting estimates of $\hat{\phi}_{1}$, which were robust to variations in the fixed parameters (Supplementary Fig. 11), are mentioned above. The best-fit parameter estimates and measures of goodness of fit are listed in Supplementary Table 5. Other parameter values employed are listed in Supplementary Table 4. (b) Pre-treatment frequency of IFN-refractory cells and the slope of the second phase decline estimated from data in two reports on patients under IFN monotherapy (closed circles: ref. 51; open circles: ref. 53) are strongly correlated (Pearson's correlation coefficient, $R=-0.44, P<0.001$ ). Using the standard model of viral dynamics, the efficacy of IFN, $\varepsilon$, and the death rate of infected cells, $\delta$, were estimated from previously published data of viral kinetics for the patients ${ }^{51,53}$. The second phase slope is the product $\delta \varepsilon$ according to the standard model. Further, $\varepsilon$ in the standard model corresponds to $\phi_{3}$ in our model, which yielded $\widehat{\phi}_{1}=1-\varepsilon$.

treatment stimulates the IFN network, which increases ISG expression and, given potent IFN effector function, clears infection. Cells in such individuals predominantly admit $Q_{3}$ at the level of IFN exposure during treatment. Such individuals are predicted to be rapid responders to IFN treatment (Fig. 7c).

When endogenous IFN production is high but IFN effector function compromised, infection is established despite stimulation of the IFN network. Here ISG levels are high before treatment (Fig. 7b), but HCV continues to thrive because of poor effector function. Cells in such individuals predominantly admit $Q_{1}$ and addition of exogenous IFN contributes little to the control of infection. Such individuals are predicted to be null responders to treatment (Fig. 7c).

When both endogenous IFN production and ISG effectiveness are low, addition of IFN may increase ISG levels but not necessarily clear HCV given the inadequate effector function. Such individuals appear as partial responders. The fraction of IFN-refractory cells is smaller in such individuals than in null responders, but treatment would fail as long as the fraction is larger than the critical value mentioned above. When both endogenous IFN production and ISG effectiveness are high, infection is likely to be spontaneously cleared; spontaneous clearance has been observed to additionally require strong cytotoxic T-cell responses ${ }^{22}$, which our study did not consider.

We note that IFN effector function is compromised at steps downstream of the step(s) in the IFN signalling network at which HCV interferes. For instance, HCV interfered above at the level of 

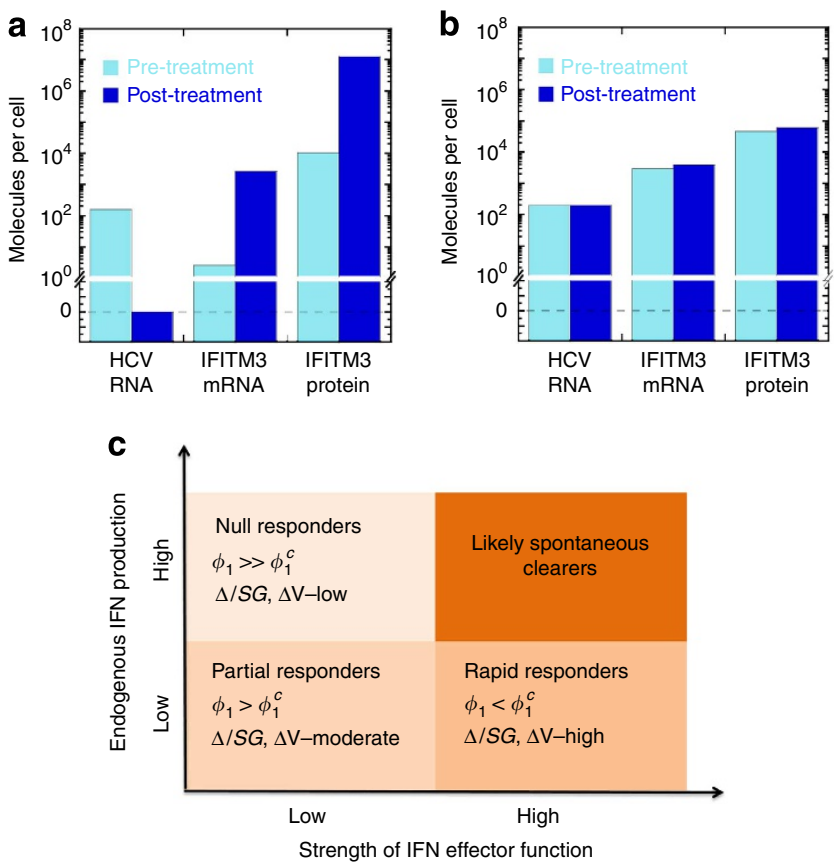

Figure 7 | Pre- and post-treatment ISG expression and treatment

response. Pre- and post-treatment steady state positive-strand HCV RNA, ISG mRNA and ISG protein expression levels, in (a), an IFN-sensitive cell with low endogenous IFN level (IFN endo $=0.05 \mathrm{U} \mathrm{ml}^{-1}$ ) and potent IFN effector function ( $\omega=0.5, \gamma=1)$, representative of responders, and $(\mathbf{b})$, an IFN-refractory cell with high-endogenous IFN level (IFN endo $_{0}=100 \mathrm{U} \mathrm{ml}^{-1}$ ) but poor effector function ( $\omega=20, \gamma=0.1$ ), representative of nonresponders. The dashed line marks zero. IFITM3 is shown as a representative ISG; other ISGs behave similarly. (c) Schematic of expected responses to IFN-based treatment in patients with different endogenous IFN production levels and potencies of IFN effector function. $\phi_{1}$ is the pretreatment frequency of IFN-refractory cells, $\phi_{1}^{c}$ is the critical pre-treatment frequency of IFN-refractory cells for successful treatment response, $\Delta V$ is the fold-decline in viral load and $\Delta / S G$ is the fold increase in ISG mRNA levels relative to their respective pre-treatment levels. $\omega$ and $\gamma$ represent the fold changes in the repression coefficients of all ISGs and the elF2B production rate constant, respectively, relative to their baseline values in Supplementary Table 3. Parameters: IFN $\mathrm{Fxo}_{\text {exo }}=100 \mathrm{U} \mathrm{ml}^{-1}$. Other parameters are listed in Supplementary Tables 1-3.

ISG translation, so that poor effector function arose from low levels of ISG proteins despite high-ISG mRNA levels. In this case, both uninfected and infected cells would display high-pretreatment ISG mRNA expression in nonresponders. If, instead, $\mathrm{HCV}$ were to predominantly inhibit the JAK-STAT pathway, low effector function would arise from low ISG mRNA expression. High-pre-treatment ISG expression in nonresponders would then be restricted to uninfected cells via paracrine signalling.

Synergy between DAAs and IFN. Finally, we examined the origin of the synergy between DAAs and IFN. DAAs such as $\mathrm{HCV}$ protease and polymerase inhibitors lower the HCV replication rate and would therefore weaken the control of HCV on the IFN network. To characterize the impact of such a DAA, we identified the steady states of the network at different (dosedependent) DAA efficacies (Fig. 8a). At low DAA efficacies, the system again exhibits the bistable behaviour observed earlier. As the efficacy increases, the HCV RNA level corresponding to $Q_{1}$ declines and that corresponding to $Q_{2}$ increases. Interestingly,
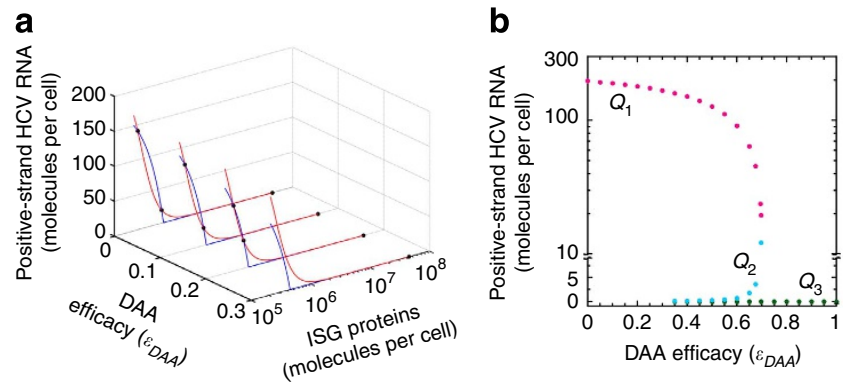

Figure 8 | Influence of DAA on IFN responsiveness. (a) Concentrationeffect curves showing steady state positive-strand HCV RNA levels for fixed ISG protein levels (blue lines) and ISG protein levels for fixed doublestranded HCV RNA levels (red lines) in the presence of a DAA at different efficacies $\left(\varepsilon_{D A A}\right)$. (b) Steady states of the network as a function of DAA efficacy. $\ln \mathbf{a}, \omega=1, \gamma=1$. $\ln \mathbf{b}, \omega=10, \gamma=0.1 . \omega$ and $\gamma$ represent the fold changes in the repression coefficients of all ISGs and the elF2B production rate constant, respectively, relative to their baseline values in Supplementary Table 3. Other parameters are listed in Supplementary Tables 1-3.

beyond a critical efficacy, the HCV-high state is rendered unsustainable and the system jumps to $Q_{3}$ where $\mathrm{HCV}$ is cleared. The DAA thus transforms a bistable cell into a cell that admits $Q_{3}$ alone. Similarly, a cell that admits $Q_{1}$ alone could be transformed into one that admits both $Q_{1}$ and $Q_{3}$ (Fig. 8b). At the cell population level, this effect of the DAA would manifest as an increase in the fraction of IFN-sensitive cells, $\phi_{3}$, at the expense of IFN-refractory cells, $\phi_{1}$, and thus result in improved treatment response.

\section{Discussion}

A hallmark of HCV infection has been its persistence in many individuals despite strong ISG expression in the liver and high doses of IFN and ribavirin ${ }^{8-10}$. What underlies this persistence has remained poorly understood. In the present study, we provide a new conceptual basis for understanding HCV persistence and response to IFN-based treatment.

Negative regulators of the IFN system, such as SOCS and USP18, as well as the recently identified IFN-associated immunosuppression have been suggested to contribute to viral persistence despite IFN stimulation ${ }^{6,20,21}$. While these mechanisms are undoubtedly important, they remain partial determinants of HCV persistence and treatment response because they do not explain when pre-treatment ISG expression would be high. Further, a direct role of HCV in suppressing IFN effector function may be important in determining treatment response because DAAs that suppress HCV replication can induce SVRs in previous null responders to PR. Our study predicts that these additional mechanisms, potentially central to $\mathrm{HCV}$ persistence and treatment response, may arise from the systems-level properties of the IFN signalling network modulated by HCV.

Our model of the IFN signalling network predicts that HCV induces bistability in the network. IFN signalling is designed to clear viral infections. HCV interferes with IFN signalling and introduces an alternative steady state where HCV persists. To our knowledge, this is the first instance identified where a virus induces bistability in an innate immune signalling network to achieve persistence. A consequence is that IFN effector function can be compromised despite high-IFN production, allowing viral persistence in the presence of high-ISG expression. Addition of exogenous IFN yields no additional antiviral effect. When more than a critical fraction of cells in an individual is refractory to 
IFN, treatment would fail. DAAs improve treatment response by suppressing HCV replication, which compromises the ability of HCV to induce bistability in the network, thereby lowering the fraction of cells refractory to IFN.

The IFN signalling network comprises a large number of molecular components that interact via multiple feedback loops ${ }^{5}$. HCV interferes with the network at several levels ${ }^{6,22}$. A complex combination of molecular players as well as parameter values determining the strengths of their interactions may thus underlie the phenotypic response of an HCV-infected cell to IFN. Currently, the limited knowledge of cell-to-cell and interpatient variations in parameter values (for example, see ref. 55) precludes a priori estimation of the prevalence of IFN-refractory cells in patients and hence the establishment of a quantitative link between network properties and viral kinetics. Nonetheless, we found that $\mathrm{HCV}$-induced bistability is a robust emergent property of the network: in addition to the translational block induced by HCV considered above, with which current in vivo data ${ }^{29}$ seems consistent, we examined other known modes of HCV interference of the IFN network and also negative regulation of IFN stimulation by ISG proteins. We found bistability in all cases (Supplementary Notes 7 and 8, Supplementary Figs 7 and 8). Bistability was also maintained under parameter regimes that mimicked the dynamic oscillations in $\mathrm{HCV}$-induced translational inhibition observed recently ${ }^{56,57}$ (Supplementary Note 9, Supplementary Fig. 9). Further, our prediction that the pretreatment prevalence of IFN-refractory cells beyond a critical fraction leads to treatment failure is independent of the origin of the bistability.

Previous models of viral kinetics have estimated the effectiveness of PR by comparisons of model predictions with viral load decline in patients ${ }^{14,46-49,58}$. Our model reproduced all the patterns of viral kinetics observed in patients by varying the fractions of cells refractory and sensitive to IFN. Alternative interpretations thus emerge of the effectiveness estimated by previous models. The extent of first phase decline, which is taken as a measure of IFN effectiveness in lowering viral production, is in our model a measure of the pre-treatment fraction of infected cells that are subsequently cured by PR. The slope of the second phase, which is taken as a combination of PR effectiveness and the loss rate of infected cells, is in our model a combination of the loss rate of infected cells and the fraction of cells refractory to PR. Larger the latter fraction, smaller the slope, and hence poorer the treatment response. The critical effectiveness of PR predicted by previous models ${ }^{47}$ for successful treatment translates to the level of PR activity necessary to render the fraction of cells refractory to PR below the critical value identified by our model. Our model thus presents an alternative yet equally consistent description of viral kinetics and one that is rooted in the properties of the IFN network.

The synergy between DAAs and IFN predicted by our model can be compromised by the emergence of resistance to DAAs, which we did not consider. Recent studies have reported the dependence of viral kinetics on SNPs even in IFN-free treatment regimens ${ }^{59,60}$ and recent trials with some two-DAA combinations without PR have been stunningly successful with $>90 \%$ SVR in previous null responders to $\mathrm{PR}^{61}$. We speculate that this success may arise from a combination of three modes of DAA action. First, the DAAs may directly suppress HCV replication. Second, they may render cells monostable and facilitate HCV clearance. Third, the resulting lower HCV levels may lead to lower IFN stimulation, which in the absence of exogenous IFN may undo the immunosuppression caused by persistent IFN signalling and effect a cure. Identifying which of these mechanisms predominates in vivo awaits new investigations and may inform future strategies of intervention.

\section{Methods}

Mathematical model of the IFN signalling network. We modelled the network (Fig. 1) based on mass action and Michaelis-Menten kinetics (Supplementary Note 1). The parameters involved (Supplementary Tables 1-3) were either obtained from the literature or estimated from analysis of independent experiments of intracellular HCV RNA kinetics (Supplementary Figs 3 and 10), ISG expression following stimulation with IFN, and suppression of HCV RNA by over-expression of ISGs (Supplementary Note 2). The fit of this model to the highly infectious HCV JFH-m4 strain can be found in Supplementary Fig. 10 and Supplementary Note 2.

Mathematical model of viral kinetics. We modified the standard model of HCV viral kinetics to incorporate subpopulations of cells with distinct phenotypic responses to IFN (Fig. 4, Supplementary Note 5). We obtained analytical approximations to the resulting first and early second phase slopes (Supplementary Note 6). The parameter values employed were obtained from the literature or estimated from fits to patient data (Supplementary Tables 4-6).

Solution of model equations and data analysis. We solved the model of the IFN signalling network and the model of viral kinetics using the Rosenbrock algorithm in Berkeley-Madonna version 8.3.18 and the ode15s routine in MATLAB. We fit the model predictions to the data using the nonlinear regression tool NLINFIT in MATLAB and estimated 95\% confidence intervals using the tool NLPARCI in MATLAB. We assessed the goodness of fits using $R^{2}$ and the Student's $t$-test on residuals. All data digitization was done using Engauge digitizer. The computer programs employed are available upon request.

\section{References}

1. Lavanchy, D. Evolving epidemiology of hepatitis C virus. Clin. Microbiol. Infect. 17, 107-115 (2011).

2. Ghany, M. G., Nelson, D. R., Strader, D. B., Thomas, D. L. \& Seeff, L. B. An update on treatment of genotype 1 chronic hepatitis C virus infection: 2011 practice guideline by the American Association for the Study of Liver Diseases. Hepatology 54, 1433-1444 (2011).

3. Kau, A., Vermehren, J. \& Sarrazin, C. Treatment predictors of a sustained virologic response in hepatitis B and C. J. Hepatol. 49, 634-651 (2008).

4. Hayes, C. N., Imamura, M., Aikata, H. \& Chayama, K. Genetics of IL28B and HCV-response to infection and treatment. Nat. Rev. Gastroenterol. Hepatol. 9, 406-417 (2012).

5. Haller, O., Kochs, G. \& Weber, F. The interferon response circuit: induction and suppression by pathogenic viruses. Virology 344, 119-130 (2006).

6. Heim, M. H. Innate immunity and HCV. J. Hepatol. 58, 564-574 (2013).

7. Micallef, J. M., Kaldor, J. M. \& Dore, G. J. Spontaneous viral clearance following acute hepatitis $\mathrm{C}$ infection: a systematic review of longitudinal studies. J. Viral Hepat. 13, 34-41 (2006).

8. Lau, D. T. et al. Innate immune tolerance and the role of Kupffer cells in differential responses to interferon therapy among patients with HCV genotype 1 infection. Gastroenterology 144, 402-413 (2013).

9. Sarasin-Filipowicz, M. et al. Interferon signaling and treatment outcome in chronic hepatitis C. Proc. Natl Acad. Sci. USA 105, 7034-7039 (2008).

10. Feld, J. J. et al. Hepatic gene expression during treatment with peginterferon and ribavirin: identifying molecular pathways for treatment response. Hepatology 46, 1548-1563 (2007)

11. Di Bisceglie, A. M. et al. Ribavirin as therapy for chronic hepatitis C. A randomized, double-blind, placebo-controlled trial. Ann. Intern. Med. 123, 897-903 (1995).

12. Fried, M. W. et al. Peginterferon alfa-2a plus ribavirin for chronic hepatitis $C$ virus infection. N. Engl. J. Med. 347, 975-982 (2002).

13. Rotman, Y. et al. Effect of ribavirin on viral kinetics and liver gene expression in chronic hepatitis C. Gut 63, 161-169 (2013).

14. Dixit, N. M., Layden-Almer, J. E., Layden, T. J. \& Perelson, A. S. Modelling how ribavirin improves interferon response rates in hepatitis $\mathrm{C}$ virus infection. Nature 432, 922-924 (2004).

15. Thomas, E. et al. Ribavirin potentiates interferon action by augmenting interferon-stimulated gene induction in hepatitis $\mathrm{C}$ virus cell culture models. Hepatology 53, 32-41 (2011).

16. Welsch, C., Jesudian, A., Zeuzem, S. \& Jacobson, I. New direct-acting antiviral agents for the treatment of hepatitis $\mathrm{C}$ virus infection and perspectives. Gut 61(Suppl 1): i36-i46 (2012).

17. Pawlotsky, J. M. Treatment failure and resistance with direct-acting antiviral drugs against hepatitis C virus. Hepatology 53, 1742-1751 (2011).

18. Muir, A. J. et al. Retreatment with telaprevir combination therapy in hepatitis $\mathrm{C}$ patients with well-characterized prior treatment response. Hepatology 54, 1538-1546 (2011).

19. McHutchison, J. G. et al. Telaprevir for previously treated chronic HCV infection. N. Engl. J. Med. 362, 1292-1303 (2010).

20. Teijaro, J. R. et al. Persistent LCMV infection is controlled by blockade of type I interferon signaling. Science 340, 207-211 (2013). 
21. Wilson, E. B. et al. Blockade of chronic type I interferon signaling to control persistent LCMV infection. Science 340, 202-207 (2013).

22. Thimme, R., Binder, M. \& Bartenschlager, R. Failure of innate and adaptive immune responses in controlling hepatitis $\mathrm{C}$ virus infection. FEMS Microbiol. Rev. 36, 663-683 (2012).

23. Schoggins, J. W. et al. A diverse range of gene products are effectors of the type I interferon antiviral response. Nature 472, 481-485 (2011).

24. Metz, P. et al. Identification of type I and type II interferon-induced effectors controlling hepatitis C virus replication. Hepatology 56, 2082-2093 (2012).

25. Raychoudhuri, A. et al. ISG56 and IFITM1 proteins inhibit hepatitis $C$ virus replication. J. Virol. 85, 12881-12889 (2011).

26. Kim, M. J. \& Yoo, J. Y. Inhibition of hepatitis C virus replication by IFNmediated ISGylation of HCV-NS5A. J. Immunol. 185, 4311-4318 (2010).

27. Han, J. Q. \& Barton, D. J. Activation and evasion of the antiviral $2^{\prime}-5^{\prime}$ oligoadenylate synthetase/ribonuclease $\mathrm{L}$ pathway by hepatitis $\mathrm{C}$ virus mRNA. RNA 8, 512-525 (2002).

28. Garaigorta, U. \& Chisari, F. V. Hepatitis C virus blocks interferon effector function by inducing protein kinase R phosphorylation. Cell Host Microbe $\mathbf{6}$, 513-522 (2009)

29. Wieland, S. et al. Simultaneous detection of hepatitis $\mathrm{C}$ virus and interferon stimulated gene expression in infected human liver. Hepatology, http:// dx.doi.org/10.1002/hep.26770 (2013).

30. Perales, C. et al. Response of hepatitis C virus to long-term passage in the presence of interferon-alpha. Multiple mutations and a common phenotype. J. Virol. 87, 7593-7607 (2013).

31. Proud, C. G. eIF2 and the control of cell physiology. Semin. Cell Dev. Biol. 16, 3-12 (2005).

32. Rowlands, A. G., Panniers, R. \& Henshaw, E. C. The catalytic mechanism of guanine nucleotide exchange factor action and competitive inhibition by phosphorylated eukaryotic initiation factor 2. J. Biol. Chem. 263, 5526-5533 (1988).

33. Dabo, S. \& Meurs, E. F. dsRNA-dependent protein kinase PKR and its role in stress, signaling and HCV infection. Viruses 4, 2598-2635 (2012).

34. Cole, J. L. Activation of PKR: an open and shut case? Trends Biochem. Sci. 32, 57-62 (2007).

35. Koev, G., Duncan, R. F. \& Lai, M. M. Hepatitis C virus IRES-dependent translation is insensitive to an eIF2alpha-independent mechanism of inhibition by interferon in hepatocyte cell lines. Virology 297, 195-202 (2002).

36. Shimoike, T., McKenna, S. A., Lindhout, D. A. \& Puglisi, J. D. Translational insensitivity to potent activation of PKR by HCV IRES RNA. Antivir. Res. 83, 228-237 (2009).

37. Terenin, I. M., Dmitriev, S. E., Andreev, D. E. \& Shatsky, I. N. Eukaryotic translation initiation machinery can operate in a bacterial-like mode without eIF2. Nat. Struct. Mol. Biol. 15, 836-841 (2008).

38. Ferrell, J. E. \& Xiong, W. Bistability in cell signaling: how to make continuous processes discontinuous, and reversible processes irreversible. Chaos 11, 227-236 (2001)

39. Das, J. et al. Digital signaling and hysteresis characterize Ras activation in lymphoid cells. Cell 136, 337-351 (2009).

40. Bhalla, U. S. \& Iyengar, R. Emergent properties of networks of biological signaling pathways. Science 283, 381-387 (1999).

41. Acar, M., Becskei, A. \& van Oudenaarden, A. Enhancement of cellular memory by reducing stochastic transitions. Nature 435, 228-232 (2005).

42. Alon, U. Network motifs: theory and experimental approaches. Nat. Rev. Genet. 8, 450-461 (2007).

43. Cheng, G., Zhong, J. \& Chisari, F. V. Inhibition of dsRNA-induced signaling in hepatitis C virus-infected cells by NS3 protease-dependent and -independent mechanisms. Proc. Natl Acad. Sci. USA 103, 8499-8504 (2006).

44. Rand, U. et al. Multi-layered stochasticity and paracrine signal propagation shape the type-I interferon response. Mol. Syst. Biol. 8, 584 (2012).

45. Levin, D., Harari, D. \& Schreiber, G. Stochastic receptor expression determines cell fate upon interferon treatment. Mol. Cell Biol. 31, 3252-3266 (2011).

46. Neumann, A. U. et al. Hepatitis C viral dynamics in vivo and the antiviral efficacy of interferon-alpha therapy. Science 282, 103-107 (1998).
47. Dahari, H., Ribeiro, R. M. \& Perelson, A. S. Triphasic decline of hepatitis C virus RNA during antiviral therapy. Hepatology 46, 16-21 (2007).

48. Rong, L., Dahari, H., Ribeiro, R. M. \& Perelson, A. S. Rapid emergence of protease inhibitor resistance in hepatitis C virus. Sci. Transl. Med. 2, 30ra32 (2010).

49. Guedj, J. \& Perelson, A. S. Second-phase hepatitis C virus RNA decline during telaprevir-based therapy increases with drug effectiveness: implications for treatment duration. Hepatology 53, 1801-1808 (2011).

50. Nowak, M. A. \& May, R. M. in Virus Dynamics: Mathematical Principles of Immunology and Virology (Oxford Univ. Press, 2000).

51. Layden, J. E. et al. First phase viral kinetic parameters as predictors of treatmen response and their influence on the second phase viral decline. J. Viral Hepat. 9, 340-345 (2002)

52. Mihm, U., Herrmann, E., Sarrazin, C. \& Zeuzem, S. Predicting response in hepatitis C virus therapy. Aliment. Pharmacol. Ther. 23, 1043-1054 (2006).

53. Ribeiro, R. M., Layden-Almer, J., Powers, K. A., Layden, T. J. \& Perelson, A. S Dynamics of alanine aminotransferase during hepatitis $\mathrm{C}$ virus treatment. Hepatology 38, 509-517 (2003).

54. Bellecave, P. et al. Cleavage of mitochondrial antiviral signaling protein in the liver of patients with chronic hepatitis $\mathrm{C}$ correlates with a reduced activation of the endogenous interferon system. Hepatology 51, 1127-1136 (2010).

55. Ireton, R. C. \& Gale, Jr M. Systems biology analyses to define host responses to HCV infection and therapy. Curr. Top. Microbiol. Immunol. 363, 143-167 (2013).

56. Garaigorta, U., Heim, M. H., Boyd, B., Wieland, S. \& Chisari, F. V. Hepatitis C virus $(\mathrm{HCV})$ induces formation of stress granules whose proteins regulate $\mathrm{HCV}$ RNA replication and virus assembly and egress. J. Virol. 86, 11043-11056 (2012).

57. Ruggieri, A. et al. Dynamic oscillation of translation and stress granule formation mark the cellular response to virus infection. Cell. Host. Microbe. 12 71-85 (2012).

58. Guedj, J. et al. Modelling shows that the NS5A inhibitor daclatasvir has two modes of action and yields a shorter estimate of the hepatitis $\mathrm{C}$ virus half-life. Proc. Natl Acad. Sci. USA 110, 3991-3996 (2013).

59. Chu, T. W. et al. Effect of IL28B genotype on early viral kinetics during interferon-free treatment of patients with chronic hepatitis C. Gastroenterology 142, 790-795 (2012)

60. Meissner, E. G. et al. IFNL4-deltaG genotype is associated with slower vira clearance in hepatitis $\mathrm{C}$, genotype-1 patients treated with sofosbuvir and ribavirin. J. Infect. Dis., http://dx.doi.org/10.1093/infdis/jit1827 (2014).

61. Hagan, L. M. \& Schinazi, R. F. Best strategies for global HCV eradication. Liver Int. 33, 68-79 (2013).

\section{Acknowledgements}

This work was supported by the Department of Science and Technology, Government of India. We thank Rahul Roy for comments.

\section{Author contributions}

P.P. and N.M.D. conceived the study and wrote the manuscript. P.P. performed the study. P.P., U.G. and N.M.D. analyzed the data, and contributed tools and reagents.

\section{Additional information}

Supplementary Information accompanies this paper at http://www.nature.com/ naturecommunications

Competing financial interests: The authors declare no competing financial interests.

Reprints and permission information is available online at http://npg.nature.com/ reprintsandpermissions/

How to cite this article: Padmanabhan, P. et al. Emergent properties of the interferon-signalling network may underlie the success of hepatitis $\mathrm{C}$ treatment. Nat. Commun. 5:3872 doi: 10.1038/ncomms4872 (2014) 